\title{
Effectiveness of Cognitive Behavioral Therapy for Caregivers of People with Dementia: A Systematic Review and Meta-Analysis
}

\author{
Oh-Young Kwon ${ }^{a}$ \\ Hyeong Sik Ahn ${ }^{b}$ \\ Hyun Jung Kim ${ }^{\text {b* }}$ \\ Kun-Woo Park ${ }^{c *}$
${ }^{a}$ Department of Neurology and Institute of Health Science, Gyeongsang National University College of Medicine, Jinju, Korea Institute for Evidence-Based Medicine and ${ }^{\mathrm{C}}$ Department of Neurology, Korea University College of Medicine, Seoul, Korea

\begin{abstract}
Background and Purpose Caregivers endure tremendous physical, emotional, and financial burdens while caring for people with dementia. The current study aimed to estimate the effectiveness of cognitive behavioral therapy (CBT) for caregivers of people with dementia (CGPWD).

Methods Studies in the MEDLINE, EMBASE, Cochrane Library, Web of Science, and SCOPUS databases were screened. Studies with a randomized controlled design and which produced CBT outcomes for CGPWD were included in this study, and we investigated these outcomes.

Results The screening of abstracts of 263 studies resulted in 12 randomized controlled trials being included in this study. The mean age of the CGPWD ranged from 51.5 to 66.2 years. The caregiver role was most frequently adopted by a female spouse or daughter. CBT for the CGPWD resulted in positive effects on various conditions, including depression, anxiety, stress, and dysfunctional thoughts. Depression was the most commonly evaluated condition, and the Center for Epidemiologic Studies Depression Scale (CES-D) was most frequently used as an inventory for depressive symptoms. The mean differences between the baseline and postintervention CES-D scores were compared between the CBT-intervention and control groups. The CES-D score decreased significantly more in the CBT-intervention group than in the control group. The difference in pooled mean differences between the two groups was -4.98 .

Conclusions CBT is an efficient intervention tool for reducing the various emotional burdens experienced by CGPWD. This meta-analysis found that CBT significantly improved the depressive symptoms of CGPWD.
\end{abstract}

Key Words dementia, caregivers, depression, cognitive therapy, behavioral therapy.
Received April 21, 2017

Revised June 23, 2017

Accepted June 23, 2017

\section{Correspondence}

Kun-Woo Park, MD, PhD

Department of Neurology,

Korea University College of Medicine,

73 Inchon-ro, Seongbuk-gu,

Seoul 02841, Korea

Tel $+82-2-920-5347$

E-mail kunu5347@gmail.com

*These authors contributed equally to this work.

\section{INTRODUCTION}

Dementia has crucial impacts on the emotional and physical situation of families and relatives who care for people with dementia (PWD). The lack of caring facilities for PWD results in most of them receiving in-home care provided by families or relatives. These caregivers of people with dementia (CGPWD) have not been appropriately trained, while they play an important role in the supervision and treatment of these patients. The inevitable progressive deterioration of symptoms of a patient gives rise to behavioral problems, which are a crucial burden that can be challenging for CGPWD. This situation commonly results in CGPWD experiencing physical, emotional, and financial burdens. ${ }^{1}$

CGPWD are usually not professional practitioners, and so they might not have received adequate training to care for PWD, while they must provide intensive at-home, long-term

(®) This is an Open Access article distributed under the terms of the Creative Commons Attribution Non-Commercial License (http://creativecommons.org/licenses/by-nc/4.0) which permits unrestricted non-commercial use, distribution, and reproduction in any medium, provided the original work is properly cited. 
care for patients, also without knowing when this caregiving will end. The burdens they are subjected to can have serious and negative consequences, particularly on their emotional and psychological health. ${ }^{2}$ Further, increased rates of morbidity, ${ }^{3}$ mortality, and depression and anxiety ${ }^{5}$ have been reported for CGPWD. An absence of relief from continuous caregiving and a lack of time for themselves are other problems faced by CGPWD. ${ }^{6}$ Several other negative consequences include impairment of quality of life, failure of goal attainment, and dysfunctional thoughts about caregiving.

Cognitive behavioral therapy (CBT) involves training individuals to develop cognitive and behavioral skills that will help them to cope with stressful and difficult situations. The cognitive skills developed through CBT are aimed at improving dysfunctional thoughts, while the behavioral skills increase pleasure when performing activities. For example, a CBT intervention has been reported to exert clinically significant effects on mood and anxiety disorders. ${ }^{7}$ CBT produces beneficial effects on various conditions and has various configurations; for example, it can be applied as either group or individual therapy, and its diverse interfaces include face-toface, web-based, and phone-based modes.

CGPWD experience pressure from the burdens of care. Although CBT is known to have positive effects on CGPWD, evidence-based assessments of its effectiveness have been inadequate. The current study applied a systemic review and meta-analysis with the aim of determining how CBT affects the perceived emotional and psychological burden of CGPWD and their coping mechanisms.

\section{METHODS}

The search strategy was established after referring to key studies; we used the following common keywords related to dementia, caregivers, and CBT: "dementia," "caregivers," "caregiver*," "care giver*”, "carer*," "cognitive therapy," "cognitive behavior* therapy," "cognitive behavior* therapies," "CBT," and "CBTs." The search strategies used are provided as an appendix to this review.

We searched the electronic databases MEDLINE, EMBASE, Cochrane Library, Web of Science, and SCOPUS from their inceptions up to November 2016. The search was performed on November 21, 2016. We also manually examined other bibliographies to avoid missing pertinent studies. No restrictions were placed on the publication date or language. The references, including articles and proceedings, were exported and managed using EndNote X7.5 (Thomson Reuters, Philadelphia, PA, USA). ${ }^{8}$

To identify studies suitable for inclusion in this review, two reviewers (O.Y. Kwon and K.W. Park) separately examined the titles and abstracts of the studies in the search results. The initial screening criteria were broad in order to ensure that all of the studies pertinent to this review could be identified. Studies were included if they met all of the following criteria: originality, randomized controlled design, inclusion of outcome evaluation of CBT for CGPWD, and inclusion of an untreated or minimally treated control group. We excluded studies where the outcomes were compared between a CBT group and groups with other specific treatment modalities. The two reviewers also independently rescreened the full-length articles or proceedings of the studies identified in the first screening. Discrepancies between the two reviewers were addressed by discussion or in consultation with a third author (H.J. Kim).

The obtained data were extracted and summarized. Study information (i.e., authors and year), population demographics of the CBT-intervention and control groups (i.e., country, age, sex, and number of subjects), type of dementia, relationship between the CGPWD and PWD, and how the control groups were recruited are summarized in Table 1. Table 2 presents summary information regarding the CBT protocol, including the interface mode, treatment paradigm, schedule of sessions, and management protocol. Evaluation time points, outcome measurement methods, and the benefits of CBT are summarized in Table 3.

Depression was the most frequently evaluated outcome in the included studies. The Center for Epidemiologic Studies Depression Scale (CES-D) was the inventory used most frequently to assess depressive symptoms. Therefore, studies using CES-D scores to assess depressive symptoms as an outcome of CBT for CGPWD were collated for the present metaanalysis. The studies that were most suitable for inclusion in the meta-analysis were those that provided CES-D scores collected immediately before and after the intervention in both the CBT-intervention and control groups. The results of these studies were amalgamated and the pooled effect sizes were obtained. Coefficients for the correlations between the preand posttest evaluations were not reported for any of the included studies, and so we set the pre-post correlation coefficient (pre-post $r$ ) as 0.7 for the meta-analysis. The overall mean difference in the CES-D scores between the CBT-intervention and control groups and the $95 \%$ confidence intervals (CIs) were calculated.

Statistical analyses were performed using Review Manager (version 5.3, Cochrane Collaboration, London, UK). The meta-analysis software also calculated the Cochrane $\mathrm{Q}$ statistics and $\mathrm{I}^{2}$, which were used to assess heterogeneity. If the $\mathrm{I}^{2}$ value was greater than $50 \%$, the included studies were considered to be substantially heterogeneous. ${ }^{9}$ If the $p$ value was less than 0.01 for the Cochrane Q statistics, the null hypothesis was rejected and the studies were considered heterogeneous. 


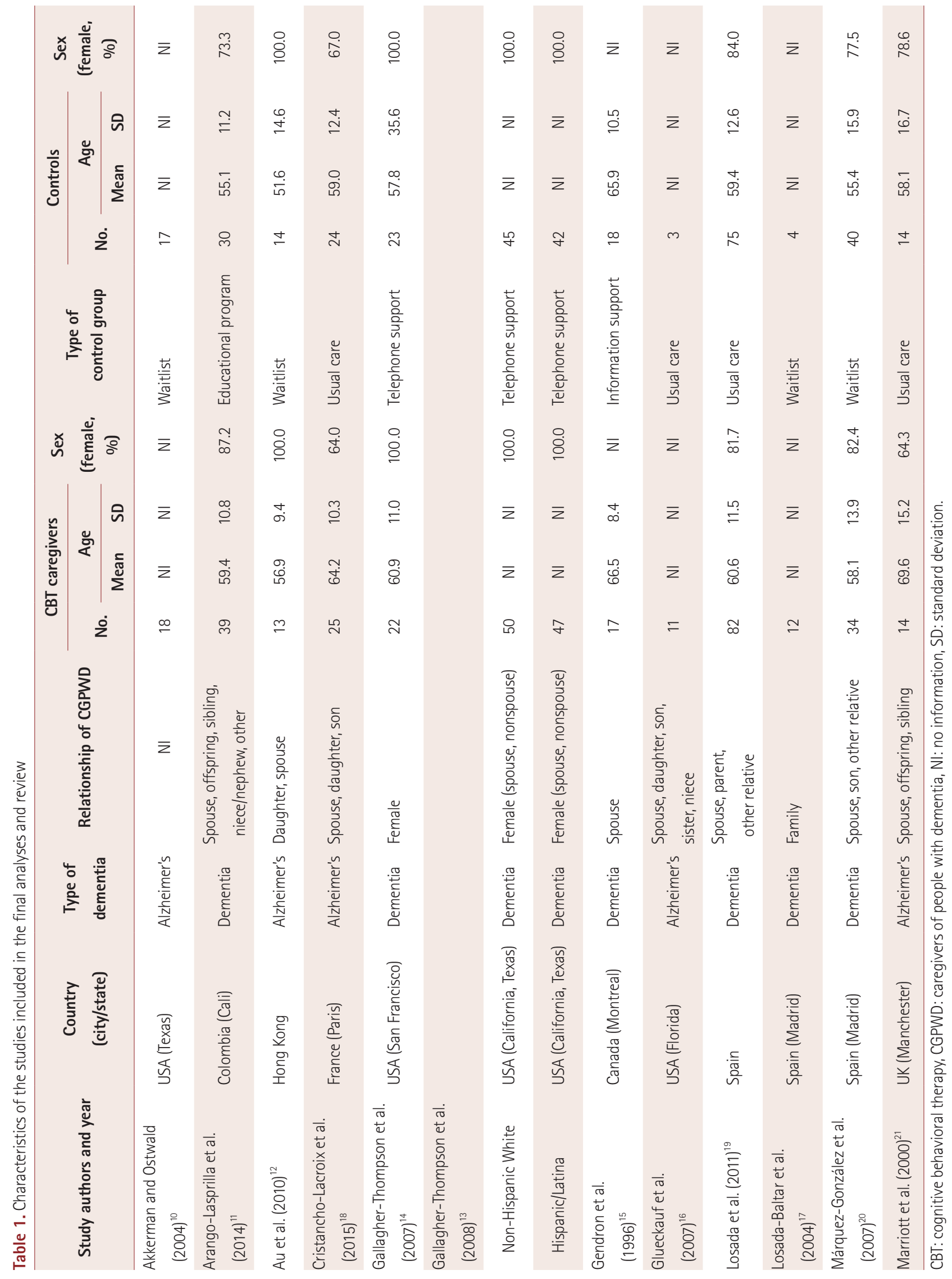




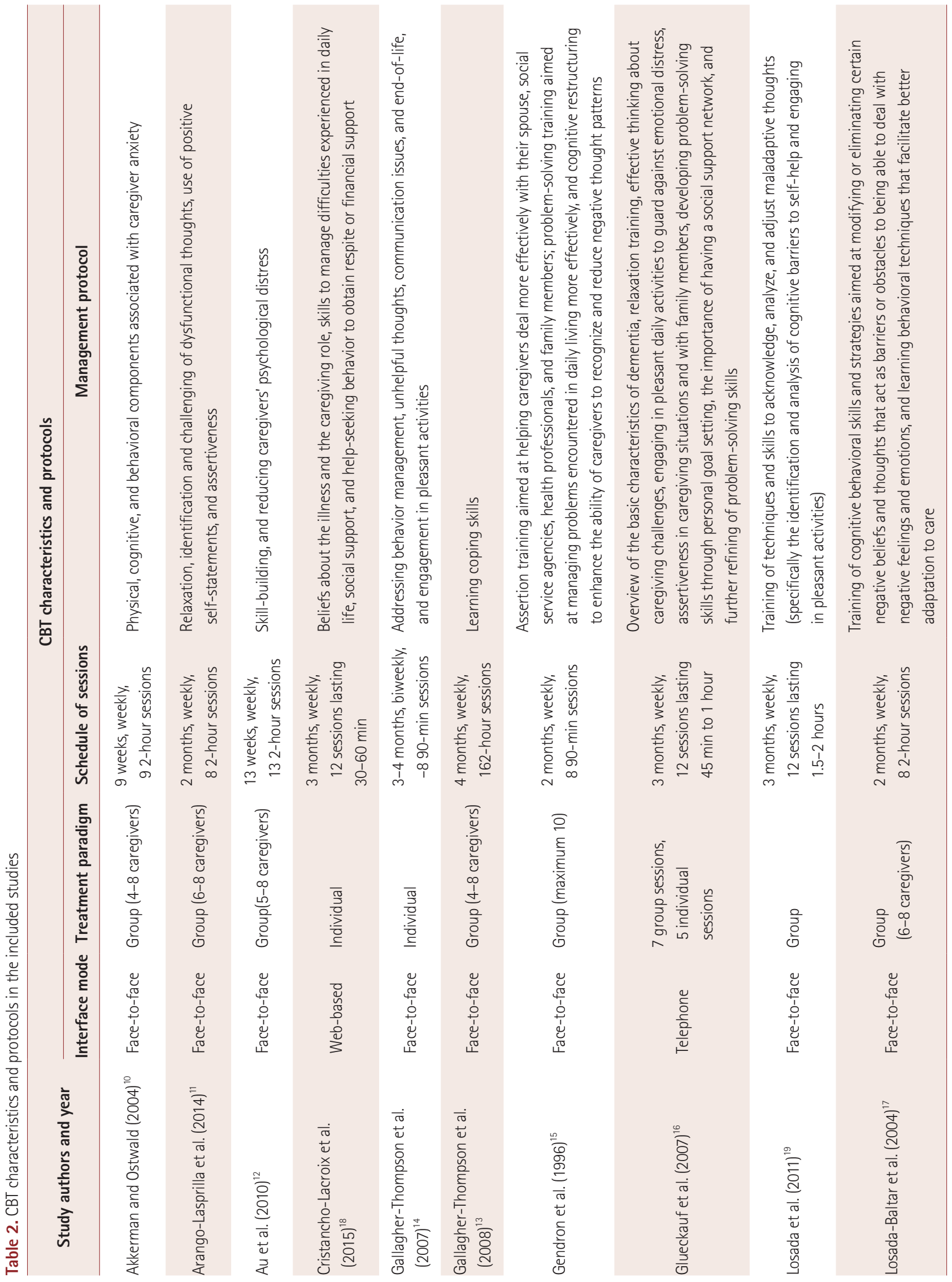




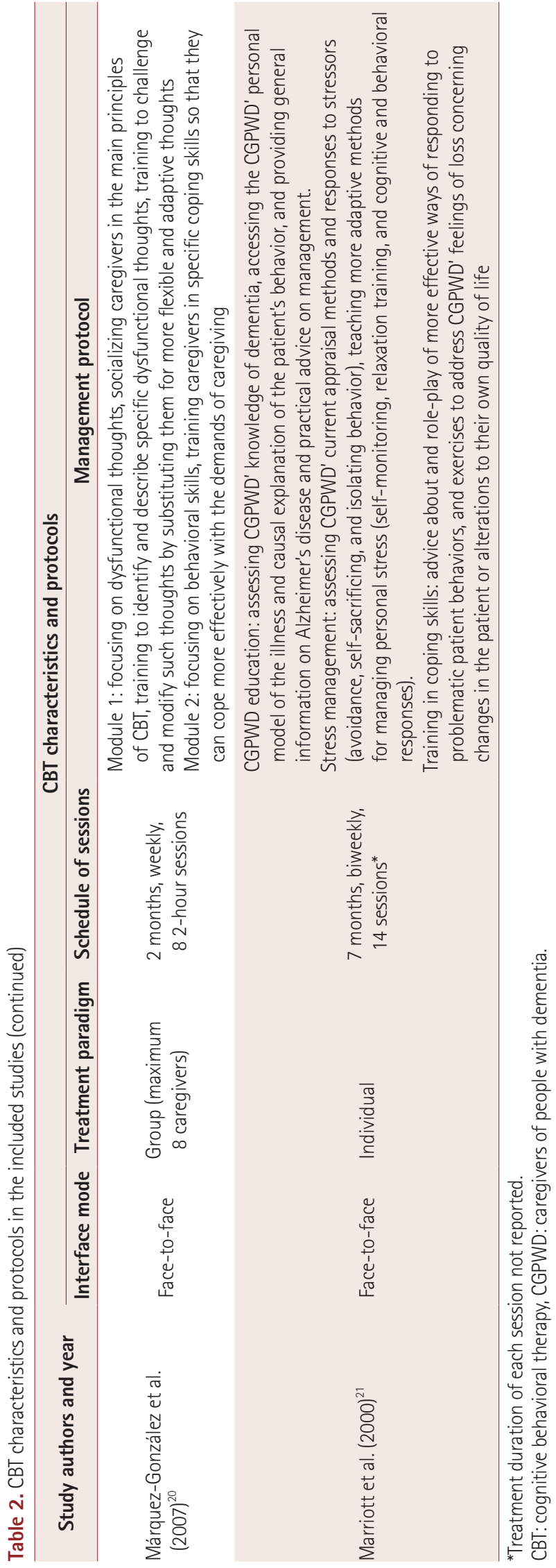

\section{RESULTS}

\section{Identification of relevant studies}

Fig. 1 shows a flow diagram of how relevant studies were identified. In total, 263 studies were identified by searching 5 databases and manually searching relevant bibliographies as follows: 37 studies from MEDLINE, 18 from EMBASE, 30 from Cochrane Library, 33 from Web of Science, 144 from SCOPUS, and 1 by manual searching. We excluded 39 duplicate studies and an additional 196 of the remaining 224 studies that did not satisfy the selection criteria. After reviewing the full texts of the remaining 28 studies, we excluded a further 16 studies based on the selection criteria for the following reasons: no outcome data $(n=7)$, no or poor randomization $(n=5)$, no control group $(n=3)$, and duplication due to incorrect publication year $(n=1)$. After reviewing the full texts, 12 studies were finally included in this study.

\section{Demographic features of caregivers of people with dementia}

The demographic features of the CGPWD are summarized in Table 1. The data on the number of CGPWD and their mean age were not reported consistently in the selected studies: they provided information on either the entire CGPWD population and/or separately on the CBT-intervention and control groups. The sample size in each of the 12 studies ranged from 14 to 170 people. Eight studies showed the mean age of the total number of CGPWD, which ranged from 51.5 to 66.2 years. ${ }^{10-17}$ The numbers of CGPWD in the CBT-intervention and control groups were provided for each of the 12 studies, and ranged from 12 to 82 and from 3 to 75, respectively. Information on the mean ages in both groups was provided for only 8 of the 12 studies. ${ }^{11,12,14,15,18-21}$ Information on the mean age of all CGPWD-in addition to those for individual groupswas also provided for four of these 8 studies. ${ }^{11,12,14,15}$ In the 8 studies for which there was information on mean ages in the groups, the mean age of the CGPWD ranged from 56.9 to 69.6 years and from 51.6 to 65.9 years in the CBT-intervention and control groups, respectively.

In all 12 included studies we were able identify the sex distribution of the total CGPWD population, including that of individual groups. ${ }^{10-21}$ Three studies ${ }^{12-14}$ involved only female CGPWD, while $65.3-87.1 \%$ of participants in the remaining 9 studies were women..$^{10,11,15-21}$ Sex ratios were provided for the total CGPWD population but not separately for the CBTintervention and control groups for 4 of the 9 studies that recruited both male and female CGPWD..$^{10,15-17}$ In the 5 studies in which sex distribution was provided for both groups, the percentage of women in the CBT-intervention and control groups ranged from 64.0 to $87.2 \%$ and from 67.0 to $84.0 \%$, 
Table 3. Outcomes of CBT for CGPWD in the studies included in the final analysis and review

\begin{tabular}{|c|c|c|c|}
\hline \multirow{2}{*}{ Study authors and year } & \multicolumn{3}{|c|}{ Outcome } \\
\hline & Evaluation time points & Measurement methods & Benefits of CBT \\
\hline $\begin{array}{l}\text { Akkerman and Ostwald } \\
(2004)^{10}\end{array}$ & Postintervention & BAl, HMMA & Anxiety \\
\hline $\begin{array}{l}\text { Arango-Lasprilla et al. } \\
(2014)^{11}\end{array}$ & $\begin{array}{l}\text { Postintervention and } \\
3 \text { months after intervention }\end{array}$ & PSS-14, PHO-9, ZBI, SWLS & $\begin{array}{l}\text { Satisfaction with life, and controlling depressive } \\
\text { symptoms }\end{array}$ \\
\hline Au et al. $(2010)^{12}$ & Postintervention & CES-D, RSCS, CWOC & $\begin{array}{l}\text { Self-efficacy for controlling upsetting thoughts, } \\
\text { handling disruptive behaviors, and using both } \\
\text { problem-focused and emotion-focused coping } \\
\text { strategies }\end{array}$ \\
\hline $\begin{array}{l}\text { Cristancho-Lacroix et al. } \\
(2015)^{18}\end{array}$ & $\begin{array}{l}\text { Postintervention and } \\
3 \text { months after intervention }\end{array}$ & $\begin{array}{l}\text { PSS-14, BDI-II, RSCS, } \\
\text { RMBPC, ZBI, NHP, VAS }\end{array}$ & Acceptance of program and expectations \\
\hline $\begin{array}{l}\text { Gallagher-Thompson et al. } \\
(2007)^{14}\end{array}$ & Postintervention & $\begin{array}{l}\text { CES-D, PSS-10, RMBPC-CB, } \\
\text { RSCS, SL-ASIA }\end{array}$ & $\begin{array}{l}\text { Being bothered by caregiving-specific stressors, } \\
\text { and depression level }\end{array}$ \\
\hline \multicolumn{4}{|l|}{$\begin{array}{l}\text { Gallagher-Thompson et al. } \\
(2008)^{13}\end{array}$} \\
\hline $\begin{array}{l}\text { Non-hispanic white } \\
\text { Hispanic/Latina }\end{array}$ & 2 months after intervention & $\begin{array}{l}\text { CES-D, PSS-10, RMBPC-CB, } \\
\text { SU0 }\end{array}$ & $\begin{array}{l}\text { Depressive symptoms, overall life stress, } \\
\text { and caregiving-specific stress }\end{array}$ \\
\hline Gendron et al. (1996) ${ }^{15}$ & $\begin{array}{l}\text { Postintervention and } 3 \text { and } \\
6 \text { months after intervention }\end{array}$ & HSC, ATQ, JCS, RAI, DAS, ZBI & Being assertive and making marital adjustments \\
\hline Glueckauf et al. $(2007)^{16}$ & Postintervention & $\begin{array}{l}\text { CES-D, CAI, CSES, ICS, } \\
\text { IFS ISS, modified CSO-8 }\end{array}$ & $\begin{array}{l}\text { Psychological distress, subjective burden, } \\
\text { and perceived self-efficacy }\end{array}$ \\
\hline Losada et al. $(2011)^{19}$ & Postintervention & CES-D, DTCQ, adapted LTS & $\begin{array}{l}\text { Dysfunctional thoughts, behavioral activation, } \\
\text { and depressive symptoms }\end{array}$ \\
\hline Losada-Baltar et al. (2004) & $\begin{array}{l}\text { Postintervention and } 3 \text { months } \\
\text { after intervention }\end{array}$ & CES-D, PSS-14, CPD, MBCL & $\begin{array}{l}\text { Perceived stress, reporting of behavioral } \\
\text { problems, and dysfunctional thoughts }\end{array}$ \\
\hline $\begin{array}{l}\text { Márquez-González et al. } \\
(2007)^{20}\end{array}$ & Postintervention & CES-D, DTCQ, MBPC, ATI & $\begin{array}{l}\text { Depressive symptoms, dysfunctional thoughts, } \\
\text { and appraisal of problem behaviors }\end{array}$ \\
\hline Marriott et al. $(2000)^{21}$ & $\begin{array}{l}\text { Postintervention and } 3 \text { months } \\
\text { after intervention }\end{array}$ & $\mathrm{GHO}-28, \mathrm{BDI}$ & $\begin{array}{l}\text { Distress, depressive symptoms, behavioral } \\
\text { disturbances, and activities }\end{array}$ \\
\hline
\end{tabular}

ATI: Assessment of Treatment Implementation, ATQ: Automatic Thoughts Questionnaire, BAI: Beck Anxiety Inventory, BDI: Beck Depression Inventory, BDI-II: Beck Depression Inventory II, CAI: Caregiver Appraisal Inventory, CBT: cognitive behavioral therapy, CES-D: Center for Epidemiologic Studies Depression Scale, CGPWD: caregivers of people with dementia, CPD: Cuestionario de Pensamientos Disfuncionales (dysfunctional thinking questionnaire), CSES: Caregiving Self-Efficacy Scale, CSQ-8: Client Satisfaction Questionnaire 8, CWOC: Chinese Way of Coping Questionnaire, DAS: Dyadic Adjustment Scale, DTCQ: Dysfunctional Thoughts about Caregiving Questionnaire, GHQ-28: General Health Questionnaire, HMMA: Hamilton Anxiety Scale, HSC: Hopkins Symptom Checklist, ICS: Issue Change Scale, IFS: Issue Frequency Scale, ISS: Issue Severity Scale, JCS: Jalowiec Coping Scale, LTS: Leisure Time Satisfaction Scale, MBCL: del Inventario de Problemas de Memoria y de Conducta, MBPC: Memory and Behavior Problems Checklist, NHP: Nottingham Health Profile, PHQ-9: Patient Health Questionnaire 9, PSS-10: the 10-item Perceived Stress Scale, PSS-14: the 14-item Perceived Stress Scale, RAI: Rathus Assertion Inventory, RMBPC: Revised Memory and Behavior Problem Checklist, RMBPC-CB: RMBPC-Conditional Bother Scale, RSCS: Revised Scale for Caregiving Self-Efficacy, SL-ASIA: Suinn-Lew Asian Self-Identity Acculturation Scale, SUQ: Skill Utilization Questionnaire, SWLS: Satisfaction With Life Scale, VAS: Visual Analog Scale, ZBI: Zarit Burden Interview.

respectively, as presented in Table $1 .^{11,18-21}$ These data show that women more commonly adopt the role of CGPWD.

The relationships of the CGPWD with PWD varied among the studies included in this review. As mentioned above, 3 studies included only female CGPWD: for one the CGPWD were described as a spouse and not a spouse of PWD, ${ }^{13}$ for another they were described as a spouse and daughter of PWD, ${ }^{12}$ and for the third they were only described as female. ${ }^{14}$ Eight of the 12 selected studies described the relationships of the CGPWD with PWD variously as spouse, daughter, sibling, son, niece/nephew, parent, or other relative; ${ }^{11,15-21}$ there was no mention of the relationship for the remaining study. ${ }^{10}$ These data suggest that female spouses or daughters most frequently adopt the role of CGPWD.

The types of dementia experienced by PWD who were nursed by CGPWD in the selected studies were not homogeneous, as indicated in Table 1 . The type of dementia was Alzheimer's disease in 5 of the 12 included studies, ${ }^{10,12,16,18,21}$ while it was described either as "dementia" or "Alzheimer's or related dementia" for the other 7 studies. ${ }^{11,13-15,17,19,20}$ 
Studies identified from the database keywords and bibliographies of relevant studies ( $n=263)$ : MEDLINE $(n=37)$, EMBASE $(n=18)$, Cochrane library ( $n=30)$, Web of science ( $n=33)$, SCOPUS ( $n=144)$, manual searching $(n=1)$

Exclude duplicate studies ( $n=39)$

Studies remaining after excluding duplicates $(n=224)$

Exclusion according to selection criteria $(n=196)$

Remaining studies ( $n=28)$, full text review

Excluded $(n=16)$

No outcome data $(n=7)$

No or poor randomization $(n=5)$

No control group $(n=3)$

Duplication due to incorrect publication year $(n=1)$

Studies included in the final analysis $(n=12)$

Fig. 1. Flow diagram for identifying relevant studies. In total, 263 studies were identified by searching 5 databases and manually searching relevant bibliographies as follows: 37 studies from MEDLINE, 18 from EMBASE, 30 from Cochrane Library, 33 from Web of Science, 144 from SCOPUS, and 1 by manual searching. We excluded 39 duplicate studies, plus an additional 196 of the remaining 224 studies that did not satisfy the selection criteria. We reviewed the full texts of the remaining 28 studies, which resulted in a further 16 studies being excluded based on the selection criteria. The reasons for exclusion of these 16 studies were no outcome data $(n=7)$, no or poor randomization $(n=5)$, no control group $(n=3)$, and duplication due to incorrect publication year $(n=1)$. After reviewing the full texts, 12 studies were finally included in this study.

The 12 selected studies had been carried out in 7 different countries (Table 1): 4 in the USA, ${ }^{10,13,14,16} 3$ in Spain, ${ }^{17,19,20}$ and 1 each in the UK, ${ }^{21}$ France, ${ }^{18}$ Canada,${ }^{15}$ Columbia,${ }^{11}$ and Hong Kong. ${ }^{12}$

\section{Methods of cognitive behavioral therapy}

Table 2 presents details of the CBT protocols used in the 12 studies. Although the detailed contents of CBT varied, the main contents included training in coping skills, modification of dysfunctional thoughts, building of a social support system, and engagement in pleasant activities. These interventions were applied both in groups and individually. Group-based CBT involved four to eight individuals in each group. Examples of programs for CBTs were psychoeducation classes on coping with caregiving, in-home behavioral management programs, and family intervention/psychoeducational inter- ventions.

Detailed information on the CBT practitioners was provided for 3 of the 12 studies: they comprised 2 clinicians with training and experience in $\mathrm{CBT},{ }^{15}$ psychologists and an occupational therapist, ${ }^{19}$ and an experienced clinical psychologist. ${ }^{21}$ We found that CBT was performed using a team approach in 9 of the 12 studies, but details of who provided CBT was not explained for these studies. ${ }^{10-14,16-18,20}$ The interface modes used for CBT varied: 10 studies $^{10-15,17,19-21}$ used a face-to-face mode while the other two used a Web-based ${ }^{18}$ and a phonebased ${ }^{16}$ mode. The CBT schedules were similar across the studies. The duration of the intervention was 2-7 months, with CBT sessions lasting 30-120 min provided weekly or biweekly (Table 2).

\section{Measurements of cognitive behavioral therapy outcomes}

Various inventories were used for measuring the outcomes following CBT in the included studies. The evaluated outcomes included depression, perceived stress, memory and behavioral problems, self-efficacy, burden of caregiving, dysfunctional thoughts about caregiving, health or symptoms, coping, and anxiety. The most commonly evaluated condition was depression, followed by perceived stress.

The inventories used are outlined in Table 3. Inventories for depressive symptoms were used in 10 studies: 7 used CES$\mathrm{D}^{12-14,16,17,19,20}$ and 1 each used the Beck Depression Inventory II (BDI-II) ${ }_{,}^{18}$ the Beck Depression Inventory (BDI), ${ }^{21}$ and Patient Health Questionnaire 9 (PHQ-9). ${ }^{11}$ Three studies used the 14-item Perceived Stress Scale ${ }^{11,17,18}$ to measure perceived stress, while another 2 studies used the 10-item Perceived Stress Scale. ${ }^{13,14}$ Memory and behavioral problems were measured using the Revised Memory and Behavior Problem Checklist ${ }^{18}$ the Revised Memory and Behavior Problem Checklist-Conditional Bother Scale, ${ }^{13,14}$ the Memory and Behavior Problems Checklist, ${ }^{20}$ and del Inventario de Problemas de Memoria y de Conducta. ${ }^{17}$ Self-efficacy was evaluated using the Revised Scale for Caregiving Self-Efficacy ${ }^{12,14,18}$ and the Caregiving Self-Efficacy Scale. ${ }^{16}$ The burden of caregiving was evaluated using the Zarit Burden Interview. ${ }^{11,15,18}$ Three studies evaluated dysfunctional thoughts about caregiving: two using the Dysfunctional Thoughts About Caregiving Questionnaire ${ }^{19,20}$ and one using del Cuestionario de Pensamientos Disfuncionales. ${ }^{17}$ The Nottingham Health Profile, ${ }^{18}$ General Health Questionnaire, ${ }^{21}$ and Hopkins Symptom Checklist ${ }^{15}$ were used to assess the health of CGPWD. The coping ability of CGPWD was evaluated using the Chinese Way of Coping Questionnaire ${ }^{12}$ and the Jalowiec Coping Scale. ${ }^{15}$

Several other inventories and scales were also used for evaluating the outcomes of the CBTs: the Beck Anxiety Inventory, 


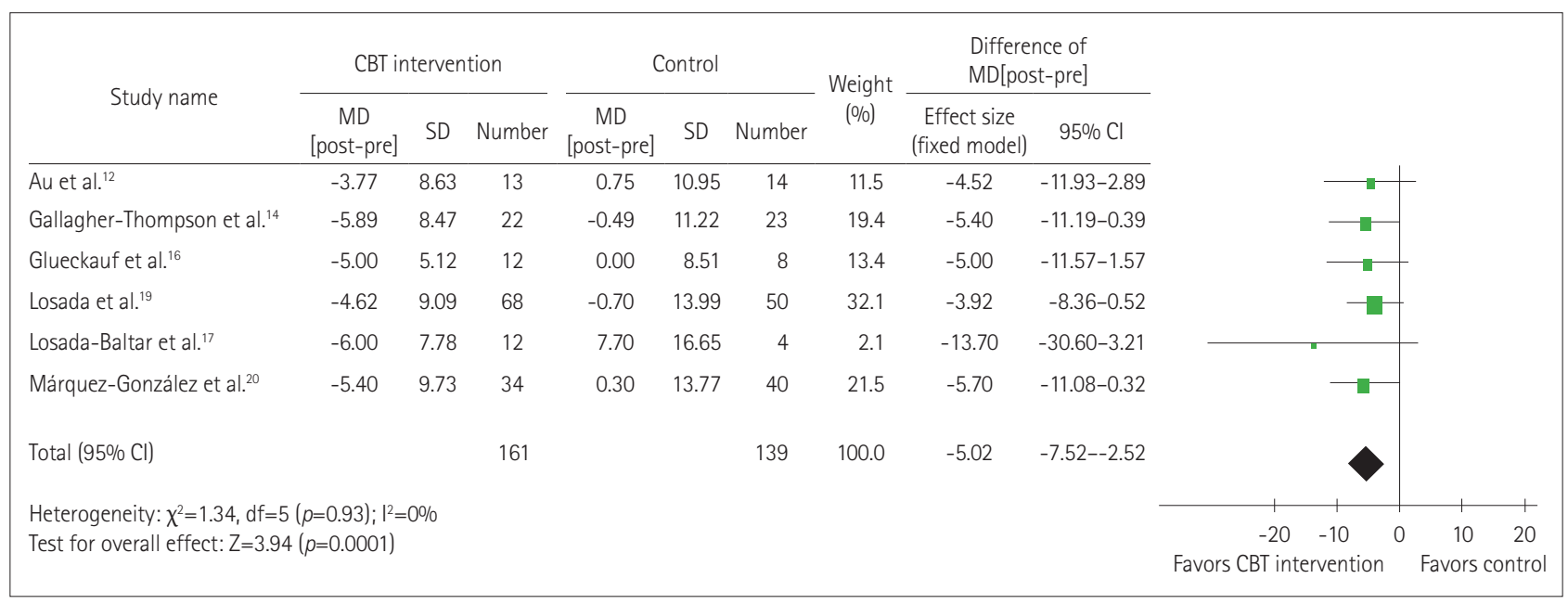

Fig. 2. Mean difference in CES-D scores between baseline and postintervention (MD[post-pre]). The mean difference was compared between CGPWD in the CBT-intervention and control groups. The mean difference was much greater in the 161 caregivers with a CBT intervention and 139 CGPWD in the control group for all 6 studies. The CES-D score was lower in the CBT-intervention group than in the control group. The pooled difference in the MD[post-pre] between the two groups in CES-D score estimated using the fixed-effects model was -5.02 (out of total score of 60), and the $95 \% \mathrm{Cl}$ was -7.52 to -2.52 . The heterogeneity among the studies was low based on the forest plot and statistical measures $(0=1.34, p=0.93$; $\left.1^{2}=0 \%\right)$. CBT: cognitive behavioral therapy, CES-D: Center for Epidemiologic Studies Depression Scale, Cl: confidence interval, CGPWD: caregivers of people with dementia, MD[post-pre]: the mean difference between the baseline and postintervention CES-D scores, SD: standard deviation.

Hamilton Anxiety Scale, Satisfaction With Life Scale, a Visual Analog Scale, Skill Utilization Questionnaire, Caregiver Appraisal Inventory, Issue Change Scale, Issue Frequency Scale, Issue Severity Scale, a modified version of the Client Satisfaction Questionnaire 8, Treatment Satisfaction, Leisure Time Satisfaction scale, Assessment of Treatment Implementation, Suinn-Lew Asian Self-Identity Acculturation Scale, Automatic Thoughts Questionnaire, Rathus Assertion Inventory, and Dyadic Adjustment Scale (Table 3).

The time points when outcomes were measured are also summarized in Table 3. All 12 studies evaluated the outcome variables at baseline, and assessments were also performed immediately after the CBT intervention in 11 of the studies; $;^{10-12,14-21}$ the postintervention evaluation was performed at 2 months after the intervention ceased in the 12th study. ${ }^{13}$ Four studies also assessed the outcomes in a follow-up evaluation at 3 months after the intervention, ${ }^{11,17,18,21}$ while 1 study assessed the outcomes in follow-up evaluations after 3 and 6 months. ${ }^{15}$

This review included studies in which the control group was a minimally treated group or an untreated group, as indicated in Table 1 . The subjects in the control groups received usual care, ${ }^{16,18,19,21}$ were on a waitlist for CBT, ${ }^{10,12,17,20}$ received telephone support, ${ }^{13,14}$ participated in educational programs, ${ }^{11}$ or received information support. ${ }^{15}$

\section{Outcomes of cognitive behavioral therapy in caregivers of people with dementia}

Table 3 summarizes the significant outcomes of each study. CBT improved the following conditions in CGPWD: depres- sion, anxiety, perceived stress, overall life stress, caregivingspecific stress, psychological distress, subjective burden, dysfunctional thoughts, and being sensitive to caregiving-specific stressors. The following characteristics of CGPWD were also enhanced: satisfaction with life, self-efficacy for controlling upsetting thoughts, perceived self-efficacy, behavioral activation, appraisal of problem behaviors, and reporting of behavioral problems. CBT exert positive effects on the following aspects of CGPWD: handling disruptive behaviors and using both problem-focused and emotion-focused coping strategies. CBT also increased the acceptance of the program and the expectations of it.

\section{Assessment of depressive symptoms}

Depressive symptoms were evaluated in 10 of the 12 studies $^{11-14,16-21}$ using the following 4 inventories: CES-D, ${ }^{12-14,16,17,19,20}$ BDI-II, ${ }^{18} \mathrm{BDI},{ }^{21}$ and PHQ-9. ${ }^{11}$

We performed a meta-analysis of the CES-D scores. In 5 of the 7 studies that used CES-D, depressive symptoms of CGPWD were evaluated at baseline and immediately after the intervention in both the CBT-intervention and control groups. ${ }^{12,14,16,19,20}$ Another study evaluated depression at three time points: baseline, immediately after the intervention, and at a 3-month follow-up. ${ }^{17}$ The 7 th study evaluated depression twice: at baseline and 2 months after the intervention. ${ }^{13}$ Therefore, as indicated in Table 3, we were able to analyze 6 studies that evaluated depression twice (at baseline and immediately after the intervention). ${ }^{12,14,16,17,19,20}$

We evaluated study heterogeneity using Cochrane Q statis- 
tics and $\mathrm{I}^{2}$ for the 6 studies that provided the CES-D scores for both the CBT-intervention and control groups. Since fewer than 10 studies were included in the evaluations, we did not create funnel plots for indicating publication bias. The risk of heterogeneity among the 6 studies was low, with a Cochrane Q statistic and $\mathrm{I}^{2}$ of $1.34(p=0.93)$ and $0 \%$, respectively. A forest plot of the 6 studies also indicated a low risk of heterogeneity (Fig. 2). The designs of the studies also were not clinically heterogeneous, and so we used a fixed-effects model to obtain the pooled estimate of CES-D scores and the $95 \% \mathrm{CI}$.

To obtain the pooled estimate, the mean difference between the baseline and postintervention CES-D scores (MD[postpre]) was compared between the CBT-intervention and control groups. We set the pre-post correlation coefficient ( $r$ ) as 0.7 , because this value was not provided for any of the 6 studies included in the meta-analysis of CES-D. Combining the data of the 6 studies included in the meta-analysis resulted in combined totals of 161 and 139 CGPWD in the CBT-intervention and control groups, respectively. The pooled $\mathrm{MD}$ [postpre] between the two groups was statistically significant ( $p=$ 0.0001). The decrease in the CES-D scores was markedly larger in the CBT-intervention group than in the control group. The pooled difference in the $\mathrm{MD}$ [post-pre] between the two groups was -5.02 , with a $95 \% \mathrm{CI}$ of -7.52 to -2.52 (Fig. 2).

One study evaluated depressive symptoms using CES-D three times: at baseline, immediately after the intervention, and at 3 months following the intervention. ${ }^{17}$ Another study assessed depressive symptoms twice: at baseline point and 2 months after the intervention. ${ }^{13}$ In the first study, the differences in $\mathrm{MD}$ [post-pre] between the CBT-intervention and control groups were - 14.00 and 4.20 immediately after and at 3 months after the intervention, respectively. This indicates that there was no sustained effect until 3 months after the treatment. ${ }^{17}$ In contrast, the difference was -5.40 at 2 months after the intervention in the second study. ${ }^{13}$ Moreover, this difference was similar to those found in other studies that evaluated depressive symptoms immediately after the intervention. This suggests that the effects of the CBT intervention are sustained for a shorter time.

We additionally investigated the 6 studies included in the meta-analysis of the CES-D scores. MD[post-pre] was available in both the CBT-intervention and control groups, and so we could obtain the intergroup difference in $\mathrm{MD}$ [post-pre], which we used to assess the effects of CBT on depression with respect to the duration, number, and treatment paradigm of the CBT sessions. The differences in $\mathrm{MD}$ [post-pre] in the 6 studies were-3.92, ${ }^{19}-4.52,{ }^{12}-5.00,{ }^{16}-5.70,{ }^{20}-5.40,{ }^{14}$ and $-13.70 .{ }^{17}$ These values indicate a remarkable improvement in depressive symptoms in 1 study, ${ }^{17}$ and that the degree of improvement was similar in the other 5 studies (Fig. 2).
We divided the studies into 2 groups based on the duration of the CBT sessions: this varied from $45 \mathrm{~min}$ to $90 \mathrm{~min}$ in 3 studies, with differences in $\mathrm{MD}$ [post-pre] of $-3.92,{ }^{19}-5.00,{ }^{16}$ and $-5.40,{ }^{14}$ and was $2 \mathrm{~h}$ in the other 3 studies, with differences of $-4.52,{ }^{12}-5.70,{ }^{20}$ and $-13.70 .{ }^{17}$ The number of CBT sessions was 12 or 13 in 3 of the 6 included studies, with differences in $\mathrm{MD}$ [post-pre] of $-3.92,{ }^{19}-4.52,{ }^{12}$ and $-5.00,{ }^{16}$ and 8 sessions in the other 3 studies, with differences of $-5.70,{ }^{20}-5.40,{ }^{14}$ and $-13.70 .{ }^{17}$ Three groups were formed when categorizing the 6 studies based on the CBT paradigm. Four studies used group therapy, with differences in $\mathrm{MD}$ [post-pre] of $-3.92,{ }^{19}-5.70,{ }^{20}$ $-4.52,{ }^{12}$ and $-13.70,{ }^{17} 1$ study used individual therapy, with a difference of $-5.40,{ }^{14}$ and 1 study used both therapy paradigms, with a difference of $-5.00 .^{16}$ Thus, we found that the CBT method did not significantly affect the outcome for depressive symptoms. The study in which there was a remarkable improvement of depressive symptoms was characterized by a longer duration, smaller number of CBT sessions, and group therapy.

\section{Study quality assessment}

The methodological quality of the studies included in the meta-analysis of the mean difference in CES-D scores was measured based on Cochrane's assessment of risk of bias. The results are summarized in Supplementary Fig. 1 (in the onlineonly Data Supplement).

\section{DISCUSSION}

CGPWD experience various burdens that can result in severe stress. They have to cope with various adverse characteristics of PWD, such as behavioral problems, inability to communicate, and feelings of loneliness. ${ }^{1,22-24}$ The associated burden and stress often result in CGPWD suffering from health problems ${ }^{25}$ including mental health problems such as depression and anxiety. ${ }^{26,27}$

CBT is a form of treatment that applies a psychoeducational approach. The aim of CBT for caregivers is to modify dysfunctional thoughts about caregiving and increase the provision of gratifying activities to care recipients. CBT for caregivers includes relaxation techniques and skills for seeking help. ${ }^{28}$ Applying CBT to CGPWD generally allows them to learn specific cognitive and behavioral skills for coping with the problems of PWD and to familiarize themselves with such skills. Accordingly, CBT will enable CGPWD to develop skills to provide quality care to $\mathrm{PWD}$, along with reducing their own psychological distress. A typical CBT program administered to CGPWD comprises the following components: introduction to the relationships between thoughts, behaviors, and emotions; educating CGPWD about identifying antecedents, beliefs, and consequences; challenging their automatic 
dysfunctional thoughts; and changing unhelpful thoughts into adaptive thoughts before the emergence of negative emotions and adverse behaviors. ${ }^{12}$

This review found that CBT exerted various positive effects on CGPWD. It improved mental problems such as depression, anxiety, stress, and dysfunctional thoughts, and also promoted factors that are needed to improve the quality of care, such as satisfaction with life, perceived self-efficacy, behavioral activation, and appraisal of problem behaviors. In addition, CBT positively influenced caregiving skills such as handling disruptive behavior and the use of coping strategies.

Previous studies have shown that the levels of depressive symptoms are significantly elevated in CGPWD. ${ }^{22,23}$ Depressive disorders are also frequent among CGPWD, with a meta-analysis finding that 176 out of 790 CGPWD (22.3\%) had a major depressive disorder. ${ }^{5}$ Some of the studies included in the present meta-analysis compared depressive disorders in CGPWD with matched controls, with 6 studies providing data on the relative risk (RR) of depressive disorder. Six studies found statistically significant $R R$ values, ranging from 2.80 to $38.68 .{ }^{5}$ Several studies included in this review evaluated depressive symptoms using one of the standardized inventories for depressive symptoms.

Depressive symptoms were the most consistently measured condition-as an outcome criterion of CBT for CGPWD-in the randomized controlled studies finally selected for inclusion in this review. CES-D was the most frequently used instrument for evaluating depressive symptoms. This 20 -item questionnaire $^{29}$ enquires about the frequency of depressive symptoms during the previous week. Depressive symptoms include affective, psychological, and somatic symptoms, and CES-D has been shown to be reliable in measuring changes in depressive symptoms in older adults ${ }^{30}$ and for cross-cultural research. ${ }^{31}$ It can therefore be a useful tool for integrating the results of research on depression in older adults from various cultural backgrounds, as in the present meta-analysis. This review found that 7 studies used CES-D, ${ }^{12-14,16,17,19,20}$ with 6 of them applying CES-D at the baseline and immediately after the end of the CBT intervention in both the CBT-intervention and control groups. ${ }^{12,14,16,17,19,20}$ The risk of heterogeneity with respect to the $\mathrm{MD}$ [post-pre] of CES-D scores among the 6 studies was low, and CBT consistently improved the depressive symptoms of CGPWD.

The 12 studies selected for inclusion in this review had been performed in 7 different countries. Only 1 study was carried out in an Asian country (Hong Kong), ${ }^{12}$ with the other 11 studies performed in Western countries. ${ }^{10,11,13-21}$ In the only Asian study, the cultural differences with the Western studies were hardly found. The mean age of the CGPWD was reported for 8 of the 12 studies, which was $51.5-66.2$ years, ${ }^{10-17}$ and is consistent with that of 54.2 years in the only Asian study. ${ }^{12}$ Six studies were included in the present meta-analysis of the CES-D score. The forest plot indicated that the Asian study was also not heterogeneous, as shown in Fig. 2. The effect size of the meta-analysis (i.e., the difference in $\mathrm{MD}$ [post-pre]) of the Asian study was -4.77 , which was similar to the pooled effect size of -4.98 . The $95 \%$ CI of the effect size of that study also overlapped those for the remaining five Western studies included in the meta-analysis. Investigating cultural differences will require more randomized controlled trials to be conducted in Asian countries.

Some limitations of the current study must be noted. First, the meta-analysis was challenging to perform due to the large diversity of the outcome measurements. Fortunately, depressive symptoms were frequently assessed as a criterion in the included studies, with CES-D frequently being used. This enabled a meta-analysis of the mean differences between the CBT-intervention and control groups in the included randomized controlled trials. Second, there was an inherent diversity in the studies due to variations in the familial relationships between the CGPWD and PWD. However, we discerned a relatively constant pattern of the relationships in the studies, which allowed a certain degree of integration of the studies. The role of CGPWD was adopted more frequently by women than by men, and the most common familial relationships of the CGPWD with PWD were a female spouse and a daughter. Finally, the CBT methods and schedules also varied between the included studies. However, the basic concept of the provided CBT was the same in all studies, and CBT was applied using similar protocols; the minor differences in the CBT protocols did not seem to hinder integration of the results of the studies.

CBT is an efficient intervention tool for reducing the emotional burdens experienced by CGPWD. This review has demonstrated that CBT can significantly improve depressive symptoms in CGPWD. In addition, the risk of heterogeneity with respect to the mean difference in the CES-D scores was low among the studies, which used CES-D to evaluate the depressive symptoms of CGPWD as a criterion of CBT. In addition to improving depressive symptoms, CGPWD experienced other benefits resulting from the CBT intervention such as positive effects on various aspects of their lives including anxiety, dysfunctional thoughts, satisfaction with life, self-efficacy, and caregiving skills. Addressing the limitations of previous studies as mentioned above should improve future research on and the clinical application of CBT for CGPWD. For example, focusing on specific family members such as the spouses or daughters of PWD could provide more reliable outcome measures in future research. Standardizing the CBT protocol could also facilitate clinical applications and 
future research studies. Further meta-analyses may yield more useful information on CBT for CGPWD with standardized assessment tools focusing on the important aspects of CGPWD outcomes.

\section{Supplementary Materials}

The online-only Data Supplement is available with this article at https://doi.org/10.3988/jcn.2017.13.4.394.

\section{Conflicts of Interest}

The authors have no financial conflicts of interest.

\section{Acknowledgements}

This work was supported by the Gyeongsang National University Fund for Professors on Sabbatical Leave in 2016.

\section{REFERENCES}

1. Yeom J, Yoo R, Bae S, Kang Y, Kim GH, Na HR, et al. Recent updates of therapeutic intervention programs for caregivers of patient with dementia: proposal of hospital-based individual therapy. Dement Neurocognitive Disord 2016;15:29-36.

2. González-Salvador MT, Arango C, Lyketsos CG, Barba AC. The stress and psychological morbidity of the Alzheimer patient caregiver. Int J Geriatr Psychiatry 1999;14:701-710.

3. Pinquart M, Sörensen S. Helping caregivers of persons with dementia: which interventions work and how large are their effects? Int Psychogeriatr 2006;18:577-595.

4. Schulz R, Beach SR. Caregiving as a risk factor for mortality: the caregiver health effects study. JAMA 1999;282:2215-2219.

5. Cuijpers P. Depressive disorders in caregivers of dementia patients: a systematic review. Aging Ment Health 2005;9:325-330.

6. Thomas P, Chantoin-Merlet S, Hazif-Thomas C, Belmin J, Montagne B, Clément JP, et al. Complaints of informal caregivers providing home care for dementia patients: the Pixel study. Int J Geriatr Psychiatry 2002; 17:1034-1047.

7. Butler AC, Chapman JE, Forman EM, Beck AT. The empirical status of cognitive-behavioral therapy: a review of meta-analyses. Clin Psychol Rev 2006;26:17-31.

8. EndNote X7.5. Philadelphia (PA): Thomson Reuters, 2016.

9. Higgins JP, Thompson SG, Deeks JJ, Altman DG. Measuring inconsistency in meta-analyses. BMJ 2003;327:557-560.

10. Akkerman RL, Ostwald SK. Reducing anxiety in Alzheimer's disease family caregivers: the effectiveness of a nine-week cognitive-behavioral intervention. Am J Alzheimers Dis Other Demen 2004;19:117-123.

11. Arango-Lasprilla JC, Panyavin I, Merchán EJ, Perrin PB, Arroyo-Anlló EM, Snipes DJ, et al. Evaluation of a group cognitive-behavioral dementia caregiver intervention in Latin America. Am J Alzheimers Dis Other Demen 2014;29:548-555.

12. Au A, Li S, Lee K, Leung P, Pan PC, Thompson L, et al. The Coping with Caregiving Group Program for Chinese caregivers of patients with Alzheimer's disease in Hong Kong. Patient Educ Couns 2010;78: 256-260.

13. Gallagher-Thompson D, Gray HL, Dupart T, Jimenez D, Thompson LW. Effectiveness of cognitive/behavioral small group intervention for reduction of depression and stress in non-hispanic white and hispanic/ latino women dementia family caregivers: outcomes and mediators of change. J Ration Emot Cogn Behav Ther 2008;26:286-303.
14. Gallagher-Thompson D, Gray HL, Tang PC, Pu CY, Leung LY, Wang $\mathrm{PC}$, et al. Impact of in-home behavioral management versus telephone support to reduce depressive symptoms and perceived stress in Chinese caregivers: results of a pilot study. Am J Geriatr Psychiatry 2007; 15:425-434.

15. Gendron C, Poitras L, Dastoor D, Pérodeau G. Cognitive-behavioral group intervention for spousal caregivers: findings and clinical considerations. Clin Gerontol 1996;17:3-19.

16. Glueckauf RL, Jeffers SB, Sharma D, Massey AJ, Davis WS, Wesley LM, et al. Telephone-based cognitive-behavioral intervention for distressed rural dementia caregivers: initial findings. Clin Gerontol 2007; 31:21-41.

17. Losada Baltar A, Izal Fernández de Trocóniz M, Montorio Cerrato I, Márquez González M, Pérez Rojo G. Differential efficacy of two psychoeducational interventions for dementia family caregivers. Rev Neurol 2004;38:701-708.

18. Cristancho-Lacroix V, Wrobel J, Cantegreil-Kallen I, Dub T, Rouquette A, Rigaud AS. A web-based psychoeducational program for informal caregivers of patients with Alzheimer's disease: a pilot randomized controlled trial. J Med Internet Res 2015;17:e117.

19. Losada A, Márquez-González M, Romero-Moreno R. Mechanisms of action of a psychological intervention for dementia caregivers: effects of behavioral activation and modification of dysfunctional thoughts. Int J Geriatr Psychiatry 2011;26:1119-1127.

20. Márquez-González M, Losada A, Izal M, Pérez-Rojo G, Montorio I. Modification of dysfunctional thoughts about caregiving in dementia family caregivers: description and outcomes of an intervention programme. Aging Ment Health 2007;11:616-625.

21. Marriott A, Donaldson C, Tarrier N, Burns A. Effectiveness of cognitive-behavioural family intervention in reducing the burden of care in carers of patients with Alzheimer's disease. Br J Psychiatry 2000; 176:557-562.

22. Schulz R, O’Brien AT, Bookwala J, Fleissner K. Psychiatric and physical morbidity effects of dementia caregiving: prevalence, correlates, and causes. Gerontologist 1995;35:771-791.

23. Schulz R, Visintainer P, Williamson GM. Psychiatric and physical morbidity effects of caregiving. J Gerontol 1990;45:181-191.

24. Russo J, Vitaliano PP, Brewer DD, Katon W, Becker J. Psychiatric disorders in spouse caregivers of care recipients with Alzheimer's disease and matched controls: a diathesis-stress model of psychopathology. $J$ Abnorm Psychol 1995;104:197-204.

25. Vitaliano PP, Zhang J, Scanlan JM. Is caregiving hazardous to one's physical health? A meta-analysis. Psychol Bull 2003;129:946-972.

26. Martire LM, Hall M. Dementia caregiving: recent research on negative health effects and the efficacy of caregiver interventions. CNS Spectr 2002;7:791-796.

27. Pinquart M, Sörensen S. Associations of stressors and uplifts of caregiving with caregiver burden and depressive mood: a meta-analysis. J Gerontol B Psychol Sci Soc Sci 2003;58:112-128.

28. Losada A, Márquez-González M, Romero-Moreno R, López J, Fernández-Fernández V, Nogales-González C. Attending to dementia caregivers diverse needs: contributions from cognitive-behavioral therapy and acceptance and commitment therapy. Clin y Salud 2015;26:41-48.

29. Radloff LS. The CES-D Scale: a self-report depression scale for research in the general population. Appl Psychol Meas 1977;1:385-401.

30. Hertzog C, Van Alstine J, Usala PD, Hultsch DF, Dixon R. Measurement properties of the Center for Epidemiological Studies Depression Scale (CES-D) in older populations. Psychol Assess 1990;2:64-72.

31. Gupta R, Yick A. Validation of CES-D scale for older Chinese immigrants. J Ment Health Aging 2001;7:257-272. 